\title{
Zum Gedenken an Professor Dr. Ansgar Belke
}

\author{
Michael Berlemann
}

Online publiziert: 6 . Oktober 2020

(C) Der/die Autor(en) 2020

Völlig überraschend verstarb das langjährige Mitglied des Editorial Boards des List Forums für Wirtschafts- und Finanzpolitik, Professor Dr. Ansgar Belke (Universität Duisburg-Essen), am 21. Juli 2020 im Alter von nur 55 Jahren in seiner Heimatstadt Münster. Sein viel zu früher Tod macht uns fassungslos und zutiefst traurig. Er hinterlässt seine Frau Claudia und seine drei Kinder Lennart, Anna und Henrik, denen unser tiefes Mitgefühl gilt.

Ansgar Belke studierte Volkswirtschaftslehre an der Westfälischen WilhelmsUniversität Münster. Im Mai 1995 promovierte er an der Ruhr-Universität Bochum bei Prof. Dr. Wim Kösters mit einer Arbeit zum Thema „Theorie und Empirie Politischer Konjunkturzyklen - Eine kritische Analyse der Zeitreihendynamik in Partisan-Ansätzen“. Seine Dissertation wurde mit dem Gebrüder-Deschauer-Preis für besonders herausragende Dissertationen ausgezeichnet. Im Jahr 2000 schloss er sein Habilitationsverfahren mit einer Arbeit zum Thema ,Wechselkursschwankungen, Außenhandel und Arbeitsmärkte: Neue theoretische und empirische Analysen im Lichte der Europäischen Währungsunion“ an der Ruhr-Universität Bochum ab und erhielt eine erweiterte Lehrbefugnis für Volkswirtschaftslehre und Quantitative Analyse (Ökonometrie). Noch im gleichen Jahr erhielt er einen Ruf auf eine Professur für Volkswirtschaftslehre, insbesondere Makroökonomie, angewandte Ökonomie und Wirtschaftspolitik an die Universität Wien. Bereits ein Jahr später wechselte er auf eine C4-Professor für Volkswirtschaftslehre, insb. Außenwirtschaft an der Universität Hohenheim. Im Jahr 2007 führte ihn sein Weg zurück in das Ruhrgebiet, wo er seit 2007 an der Universität Duisburg-Essen eine W3-Professor

\footnotetext{
M. Berlemann $(\bowtie)$

Fakultät Wirtschafts- und Sozialwissenschaften, Helmut-Schmidt-Universität Hamburg, Holstenhofweg 85, 22043 Hamburg, Deutschland

E-Mail: Michael.Berlemann@hsu-hh.de
} 
für Volkswirtschaftslehre, insbesondere Makroökonomik innehatte. Seit 2012 war er (ad personam) Jean Monnet Professor an der Universität Duisburg-Essen.

Die Forschungsgebiete von Ansgar Belke lagen im Bereich der Geld- bzw. Währungstheorie und -politik, der Europäischen Integration, der internationalen Makroökonomik, der Transformationsökonomik und der Finanzmarktanalyse. Methodisch arbeite er vor allem mit den modernen Methoden der Makroökonometrie. Dabei war Ansgar Belke ein außergewöhnlich aktiver Forscher, dessen Publikationsliste unfassbare 664 wissenschaftliche Beiträge umfasst. Darunter sind zahlreiche Fachartikel in internationalen Fachzeitschriften mit hoher Reputation. Daneben hat er aber auch zahlreiche Monographien, didaktische Beiträge und Aufsätze zur Politikberatung verfasst. Seine herausragende Forschungsleistung wird auch dadurch dokumentiert, dass Ansgar Belke in verschiedenen Forschungsrankings ausgezeichnete Plätze belegte. So wurde er im Handelsblatt-Ranking des Jahres 2017 in der Kategorie des wissenschaftlichen Lebenswerks auf Platz 72 deutschsprachiger Ökonomen und damit unter den besten $2 \%$ geführt. Zudem belegte er im Ranking der aktuell forschungsaktivsten deutschsprachigen Forscher im Jahr 2017 gar den 19. Platz. Seine Forschung wird von der wissenschaftlichen Gemeinschaft in hohem Maße wahrgenommen, was in einem h-Index von 22 (Scopus) nachdrücklich zum Ausdruck kommt.

Ansgar Belke hat sich aber nicht nur mit der Gewinnung neuer Forschungsergebnisse beschäftigt, sondern war auch stets sehr daran interessiert, diese Ergebnisse in die praktische Wirtschaftspolitik zu tragen. So war er z. B. von 2009 bis 2014 Mitglied des „Monetary Expert Panels“ des Europäischen Parlaments, externer Berater des DG ECFIN der Europäischen Kommission und des European Union Committee des House of Lords (UK). Zudem führte er eine Vielzahl von Drittmittelprojekten durch, zum Teil in Kooperation mit Wirtschaftsforschungsinstituten und Zentralbanken.

Es war Ansgar Belke immer ein Anliegen, den wissenschaftlichen Nachwuchs zu fördern. Dies kommt nicht nur (aber doch sehr nachdrücklich) darin zum Ausdruck, dass er nicht weniger als 65 Doktoranden und Doktorandinnen bei der Anfertigung ihrer Dissertationen betreute. Einige seiner wissenschaftlichen Schützlinge sind dabei auch der Wissenschaft treu geblieben und haben heute selbst Professuren inne. Trotz seines immensen Arbeitspensums stand er dem wissenschaftlichen Nachwuchs stets mit Rat und Tat zur Seite.

Es gibt nur wenige Forscher die national und international ähnlich gut vernetzt sind wie Ansgar Belke es war. So war er z.B. Mitglied der Ausschüsse für Wirtschaftspolitik und für Außenwirtschaftstheorie und -politik der Gesellschaft für Wirtschafts- und Sozialwissenschaften (Verein für Socialpolitik), Mitglied des Vorstands des Arbeitskreises Europäische Integration (AEI) und des Wissenschaftlichen Direktoriums des „Instituts für Europäische Politik“ (IEP). Gleichzeitig war er Associate Senior Research Fellow am Centre for European Policy Studies (CEPS), Senior Research Fellow am Centre for Data Analytics for Finance and Macroeconomics (DAFM) an der King's Business School London, Präsident der European Economics and Finance Society (EEFS) und Mitglied des Executive Committee der International Atlantic Economic Society (IAES), um nur einige wenige seiner Mitgliedschaften und Ämter zu nennen. 
Als sei es nicht genug, hat sich Ansgar Belke auch stets für wissenschaftliche Zeitschriften engagiert und wirkte in zahlreichen Herausgebergremien mit. Seit 2013 war er Mitglied des Editorial Boards des List Forums für Wirtschafts- und Finanzpolitik und hat in dieser Funktion wertvolle Impulse für die Fortentwicklung der Zeitschrift gegeben. Die Herausgeber des List Forums werden Ansgar Belke sehr vermissen.

Mit Ansgar verliert das List Forum und die wissenschaftliche Gemeinschaft nicht nur einen herausragenden Wissenschaftler, sondern auch einen unglaublich netten und hilfsbereiten Menschen. Ich habe Ansgar in meiner Studienzeit an der RuhrUniversität Bochum kennen und als sehr engagierten, hilfsbereiten und ökonometrisch außergewöhnlich sachkundigen Übungsleiter schätzen gelernt. Über die Jahre hinweg sind wir stets in engem Kontakt geblieben. Gerade in meiner Promotionsund Habilitationszeit war mir Ansgar stets ein wertvoller Ratgeber und hat mehrfach als Gutachter für mich fungiert. Aber auch danach haben sich unsere Wege sehr häufig gekreuzt, sowohl beruflich als auch privat. Die Gewissheit, dass dies in Zukunft nicht mehr möglich ist, erfüllt mich mit tiefer Trauer.

Funding Open Access funding enabled and organized by Projekt DEAL.

Open Access Dieser Artikel wird unter der Creative Commons Namensnennung 4.0 International Lizenz veröffentlicht, welche die Nutzung, Vervielfältigung, Bearbeitung, Verbreitung und Wiedergabe in jeglichem Medium und Format erlaubt, sofern Sie den/die ursprünglichen Autor(en) und die Quelle ordnungsgemäß nennen, einen Link zur Creative Commons Lizenz beifügen und angeben, ob Änderungen vorgenommen wurden.

Die in diesem Artikel enthaltenen Bilder und sonstiges Drittmaterial unterliegen ebenfalls der genannten Creative Commons Lizenz, sofern sich aus der Abbildungslegende nichts anderes ergibt. Sofern das betreffende Material nicht unter der genannten Creative Commons Lizenz steht und die betreffende Handlung nicht nach gesetzlichen Vorschriften erlaubt ist, ist für die oben aufgeführten Weiterverwendungen des Materials die Einwilligung des jeweiligen Rechteinhabers einzuholen.

Weitere Details zur Lizenz entnehmen Sie bitte der Lizenzinformation auf http://creativecommons.org/ licenses/by/4.0/deed.de. 